\title{
Asymmetric Binary Frequency Shift Keying and its Frequency Attribute
}

\author{
Shikai Zhang1, a, Jianli Jin 2 ,b \\ ${ }^{1}$ School of Information and Engineering, Southeast Univerisity, 210096, China \\ ${ }^{2}$ School of Mechatronics Engineering, Henan University of Science and Technology, Luoyang 471003, China \\ aemail: zhangsknj@163.com
}

Keywords: ABFSK, Quasi-continuous phase, Time domain, Frequency domain, Occupied bandwidth

\begin{abstract}
An innovative asymmetric binary frequency shift keying (ABFSK) modulation format is presented and studied both in the time domain and frequency domain. First, the modulation function is demonstrated, it shows a quasi-continuous phase attribute and an ability of high speed data modulation. Second, the domain attribute is studied; it has a high degree of energy centralization. Thus, a narrow occupied bandwidth can be achieved after a proper filtering. Then, the finite spectrum can be conserved to improve spectrum efficiency.
\end{abstract}

\section{Introduction}

Now wireless devices are widely used. It seems that wireless application have been being penetrating into all aspects of our daily life. The demanding is higher than any other periods. Whereas the media used for wireless propagation is limited, it is valuable as other non-renewable resources. So it must be used carefully $[1,2]$, and how to improve use efficiency is an important and time compelling research topic. Different modulation methods have been studied such as FQPSK, OFDM and MIMO etc. Cognitive Radio (CR) is especially a new thought about dynamic use of spectrum resource [3]. All these approaches are based the traditional modulation techniques. So if the key information bearing approach can be improved, the frequency spectrum efficiency will be enhanced fundamentally. Traditionally, the modulation is symmetric or balanced, so that the carrier and line spectrum is reduced as much as possible. Then it is recognized the information energy is maximized, while, which leads to a proprietary band licensed and occupied. This is maybe the reason why spectrum scarce is problem people are facing. In fact, spectrum can be used in another fashion, but this depends on the new radio disturbed way that information bears on the media. As an innovative modulation method, asymmetric binary frequency shift keying (ABFSK) is proposed. Being different the traditional modulation approach, ABFSK tries to disturb the carrier as little as possible. So the energy of the modulated signal is very much, even ultra, centered, only a small fraction of energy is distributed on either side of the centre frequency and the occupied bandwidth is reduced to a large degree. In the article, modulation method will be given, and time domain and frequency domain characteristics will be studied.

\section{Asymmetric Binary Frequency Shift Keying}

Modulation Function. ABFSK is defined as [4]:

$$
\begin{gathered}
g_{0}(t)= \begin{cases}\cos (2 \pi f t) & 0 \leq t<\tau_{1}, 0 \leq \tau_{1} \leq T_{s}-\tau \\
\cos (2 \pi(f+\Delta f) t) & \tau_{1} \leq t<\tau_{2}, 0 \leq \theta, \theta_{0} \leq \pi \\
\cos (2 \pi f t) & \tau_{2} \leq t<T_{s}\end{cases} \\
g_{1}(t)=\cos (2 \pi f t)
\end{gathered}
$$


$g_{0}(t)$ represents the digital " 0 ", and $g_{1}(t)$ represents the digital " 1 ". Only a small fraction of $g_{0}(t)$ is modulated, so $g_{0}(t)$ and $g_{1}(t)$ shows "asymmetry". $\theta_{0}$ is constant, and $\theta_{0}=0$ is preferred. $T_{S}$ is the bit period, and $\tau=\tau_{2}-\tau_{1}$ is the modulation duration, which takes value between 0 and $T$. $\theta$ and $\tau$ comprise the complex modulation index, $\tau / T$ can is the modulation duty, and it represents the degree of asymmetry between $g_{0}(t)$ and $g_{1}(t)$. If $\Delta f=0$, the modulated result is pure sinusoidal wave. For other values, it is a kind of asymmetric frequency modulation. $\Delta f$ can be taken two or more different values, such as $\Delta f=\Delta f_{1}$ or $\Delta f=\Delta f_{2}$ chosen, then the modulated signal includes three discrete frequency in the time domain, $f_{c}, f_{c}+\Delta f$ and $f_{c}-\Delta f$. An example of ABFSK is plotted in Fig.1.

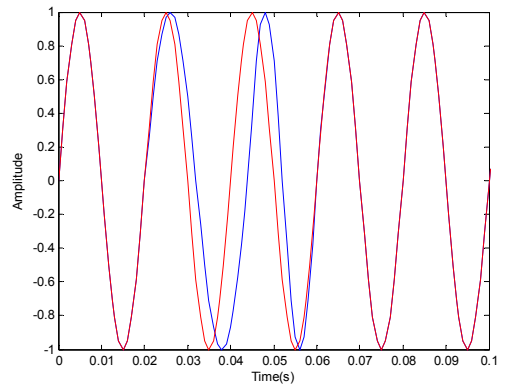

Fig. 1 waveforms of ABFSK when $T=0.1 s, \tau_{1}=0.02 s, \tau_{2}=0.06 s, \tau=0.04 s$ and $\theta_{0}=0$

Another Form. As for ABFSK modulation format, for (1), $\Delta f=\Delta f_{1}$ and $\Delta f=\Delta f_{2}$ are chosen, and, at the same time, the following relation is satisfied $1 /\left(1+\Delta f_{1}\right)+1 /\left(1+\Delta f_{2}\right)=2 / f_{C}$. Under all these assumptions, ABFSK is expressed as in another form:

$$
\begin{gathered}
g_{0}(t)= \begin{cases}\cos \left(2 \pi\left(f+\Delta f_{1}\right) t\right) & 0 \leq t<\tau_{1} \\
\cos \left(2 \pi\left(f+\Delta f_{2}\right) t\right) & \tau_{1} \leq t<\tau_{2}, \\
\cos (2 \pi f t) & \tau_{2} \leq t<T_{s}\end{cases} \\
\left\{\begin{array}{l}
g_{1}(t)= \begin{cases}\cos \left(2 \pi\left(f+\Delta f_{2}\right) t\right) & 0 \leq t<\tau_{1} \\
\cos \left(2 \pi\left(f+\Delta f_{1}\right) t\right) & \tau_{1} \leq t<\tau_{2}, \\
\cos (2 \pi f t) & \tau_{2} \leq t<T_{s}\end{cases} \\
g_{1}(t)=\cos (2 \pi f t)
\end{array}\right.
\end{gathered}
$$

Here, $\alpha=\Delta f / f_{c}$, which represents the difference of the modulated waveforms or the modulation degree. $\tau_{2}=2 / f_{c}, \tau_{1}=1 /\left(f_{c}+\Delta f_{1}\right)$. For $\alpha=0$, the waveform is two cycles of sine wave, and there are no difference between $g_{0}(t)$ and $g_{1}(t)$, which has the narrowest frequency spectrum, it is one extreme case; with $\alpha$ increasing, difference of the two signaling signal is larger and larger. One cycle is enlarged and the other is compressed. At the other extreme case, $\tau_{1}=\tau_{2}$, which reaches the other extreme case of modulation. Certainly, this also causes the largest spectrum spreading.

\section{Frequency Domain Attributes}

Expression for PSD. For ABFSK modulation symbol $g_{0}(t)$ and $g_{1}(t)$, If $g_{0}(t)$ and $g_{1}(t)$ are viewed as two states, the modulated signal can be expressed as a state transfer diagram. If the current signal is independent of the previous one, then, the general PSD can be given as the following equation [5],

$$
G_{s}(f)=\frac{1}{T_{s}}\left[\sum_{i=1}^{M} P_{i}\left|G_{i}(f)\right|^{2}-\left|\sum_{i=1}^{M} P_{i} G_{i}(f)\right|^{2}\right]+\frac{1}{T_{s}^{2}} \sum_{m}\left|\sum_{i=1}^{M} P_{i} G_{i}\left(\frac{m}{T_{s}}\right)\right|^{2} \delta\left(f-\frac{m}{T_{s}}\right)
$$


Where, $G_{i}(f), i=1,2 \cdots M$ is the Fourier transform of $g_{i}(t) \cdot P_{i}, i=1,2 \cdots M$ is the corresponding probability of $g_{i}(t), M$ represents the size of the symbol set.

PSD for ABFSK. With the discussion above, Substitute Fourier transforms of $g_{0}(t)$ and $g_{1}(t)$ above into (3), we achieve the PSD formula of ABFSK, the plots are shown in Fig.2, and the corresponding PSD simulated given in Fig.3.

$$
\begin{aligned}
G_{s}(f)= & \frac{1}{T_{s}}\left[\sum_{i=1}^{2} \frac{1}{2} \mid \tau \tau_{1} S a\left(\pi f \tau_{1}\right)\left[\delta\left(f-f_{1}\right)+\delta\left(f+f_{1}\right)\right] \exp \left(-j \pi f \tau_{1}\right)+\left(\tau_{2}-\tau_{1}\right) S a\left(\pi f\left(\tau_{2}-\tau_{1}\right)\right)\left[\delta\left(f-f_{2}\right)+\delta\left(f-f_{2}\right)\right] \exp \left(-j \pi f\left(\tau_{2}+\tau_{1}\right)\right)\right. \\
& +\left.\left(T_{s}-\tau_{2}\right) S a\left(\pi f\left(T_{s}-\tau_{2}\right)\right)\left[\delta\left(f-f_{c}\right)+\delta\left(f+f_{c}\right)\right] \exp \left(-j \pi f\left(T_{s}+\tau_{2}\right)\right)\right|^{2}-\left|\sum_{i=1}^{2} \frac{1}{2}\right| \tau_{1} S a\left(\pi f \tau_{1}\right)\left[\delta\left(f-f_{1}\right)+\delta\left(f+f_{1}\right)\right] \exp \left(-j \pi f \tau_{1}\right) \\
& \left.+\left(\tau_{2}-\tau_{1}\right) S a\left(\pi f\left(\tau_{2}-\tau_{1}\right)\right)\left[\delta\left(f-f_{2}\right)+\delta\left(f-f_{2}\right)\right] \exp \left(-j \pi f\left(\tau_{2}+\tau_{1}\right)\right)+\left.\left(T_{s}-\tau_{2}\right) S a\left(\pi f\left(T_{s}-\tau_{2}\right)\right)\left[\delta\left(f-f_{c}\right)+\delta\left(f+f_{c}\right)\right] \exp \left(-j \pi f\left(T_{s}+\tau_{2}\right)\right)\right|^{2}\right] \\
& +\frac{1}{T_{s}^{2}} \sum_{m} \sum_{i=1}^{2} \frac{1}{2} \mid \tau_{1} S a\left(\pi f \tau_{1}\right)\left[\delta\left(f-f_{1}\right)+\delta\left(f+f_{1}\right)\right] \exp \left(-j \pi f \tau_{1}\right)+\left(\tau_{2}-\tau_{1}\right) S a\left(\pi f\left(\tau_{2}-\tau_{1}\right)\right)\left[\delta\left(f-f_{2}\right)+\delta\left(f-f_{2}\right)\right] \exp \left(-j \pi f\left(\tau_{2}+\tau_{1}\right)\right) \\
& +\left.\left(T_{s}-\tau_{2}\right) S a\left(\pi f\left(T_{s}-\tau_{2}\right)\right)\left[\delta\left(f-f_{c}\right)+\delta\left(f+f_{c}\right)\right] \exp \left(-j \pi f\left(T_{s}+\tau_{2}\right)\right)\right|^{2} \delta\left(f-\frac{m}{T_{s}}\right)
\end{aligned}
$$

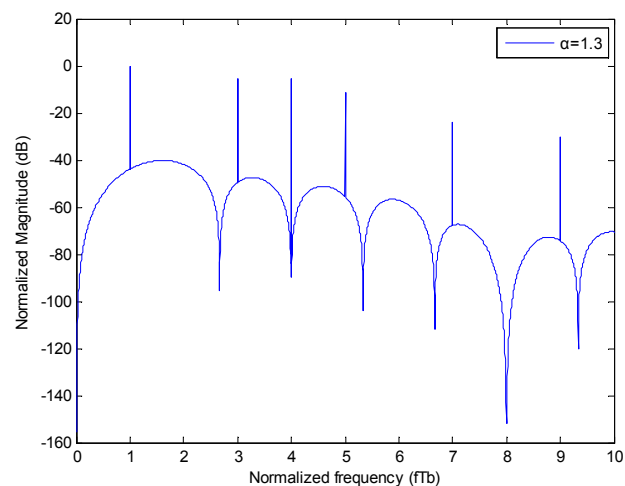

(a)

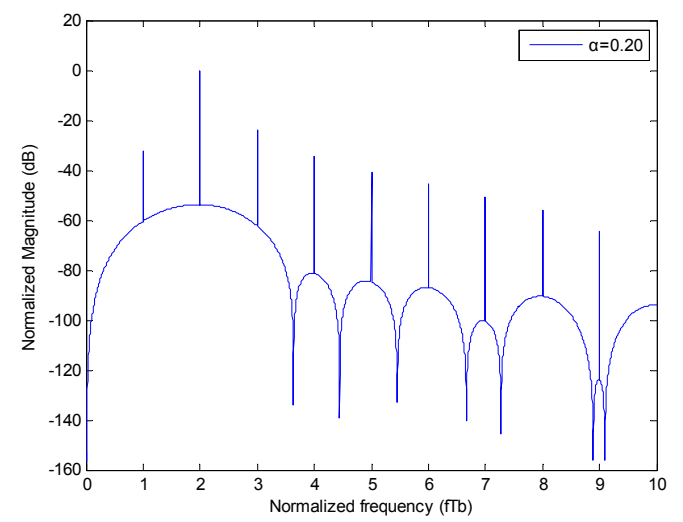

(c)

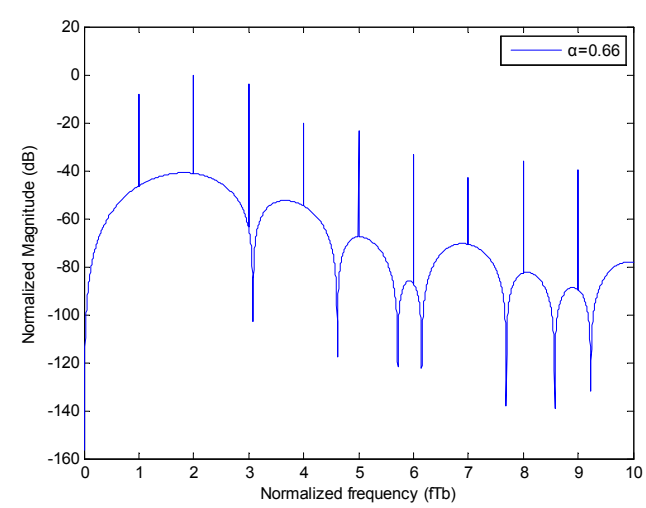

(b)

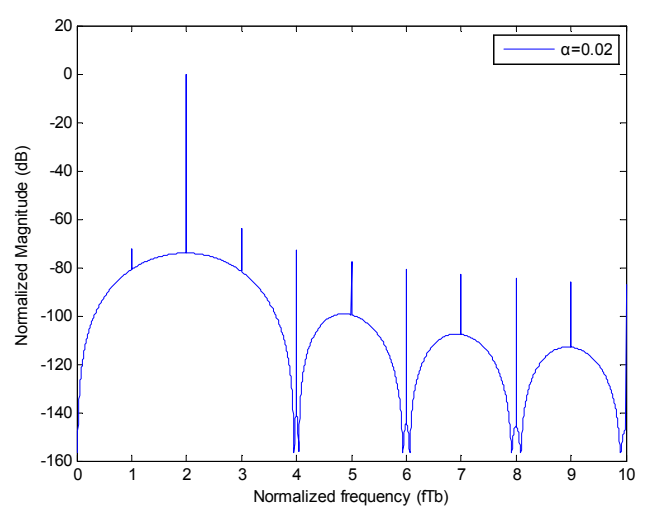

(d)

Fig.2 PSD calculated for ABFSK
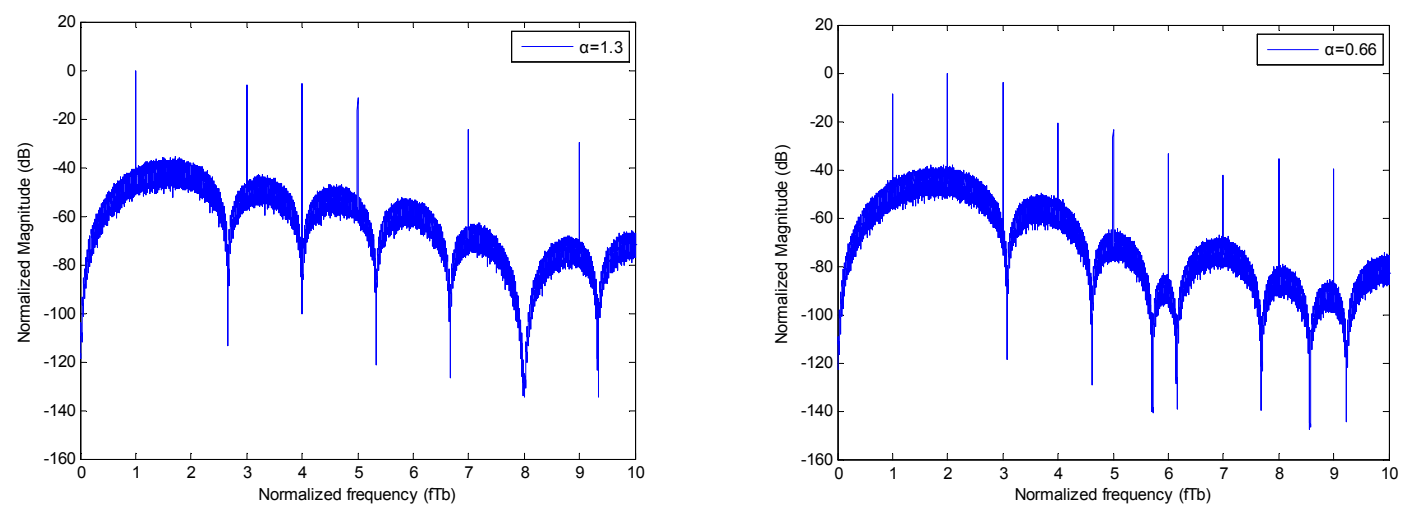
(a)

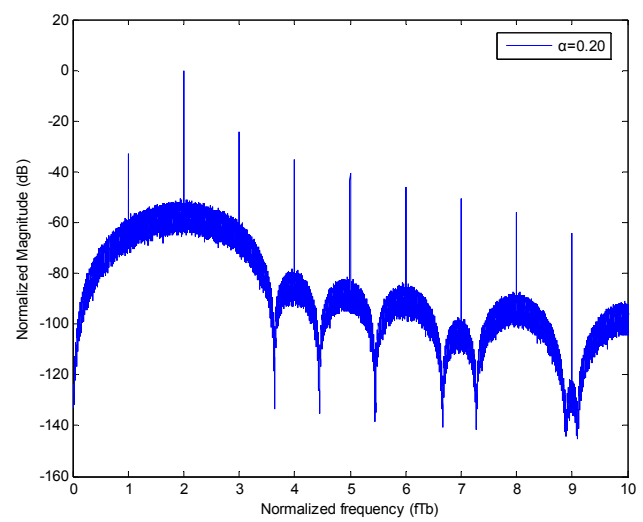

(c) (b)

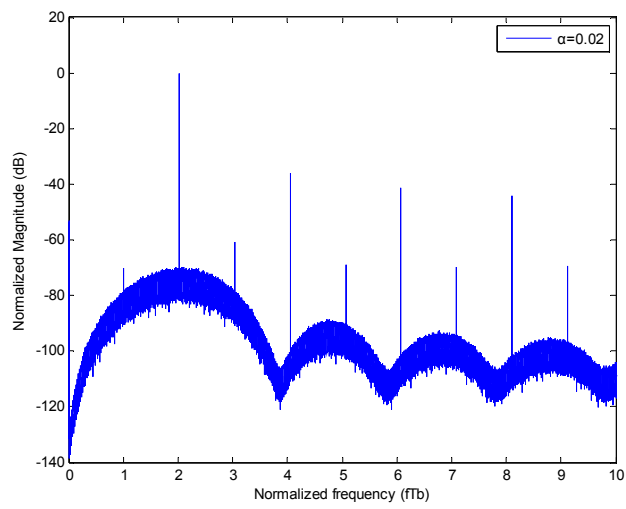

(d)

Fig. 3 PSD simulated for ABFSK

From Fig.2, with $\alpha$ decreasing from $\alpha=1.3$ to 0.02 , the power level of the continuous spectrum becomes lower and lower from about $-40 \mathrm{~dB}$ at the beginning $\alpha=1.3$ down to about $-80 \mathrm{~dB}$ at $\alpha=0.02$. More and more power concentrates to the carrier. With $\alpha$ decreasing, the main lobe is wider and wider, when $\alpha=1.3$, it about 2.5 times symbol rates, and till $\alpha=0.02$, it approaches 4 times symbol rates. At the same time, with $\alpha$ decreasing, the side lobe attenuates faster and faster. When $\alpha$ is small enough, the side lobes can be omitted shown in Fig.3 (d). Corresponding to the theoretical calculation, the spectrum simulated is shown in Fig.3, which is consistent with the theory value and shows the same changing laws. Besides the continuous main lobe and the carrier line frequency, there are two line spectra equally spaced on either side of the carrier frequency by the data rates. For ABFSK, one bit consists of two carrier cycles, so the data rates can reach the high rate of half carrier frequency; obviously it is a high speed modulation methods.

\section{Summary}

Based on the asymmetric coding method, ABFSK modulation format is presented and studied both in the time domain and frequency domain. In the time domain, the waveform is quasi-continuous. The phase is continuous but the waveform is not smooth at the transition point between sine cycle and the modulated cycle. At the extreme case, only two cycles is used to transmit on symbol, so it is a high speed modulation format. In the frequency, it has a good energy distribution. The energy is highly concentrated near the carrier. After proper filtering, it has a narrow occupied bandwidth. So we can achieve high bandwidth efficiency and conserve the finite spectrum resources.

\section{Acknowledgment}

This work is supported by National Natural Science Foundation of China under Grant number 61001102. And the author would like to thank the funding and subsidization.

\section{References}

[1] S.K. Zhang, UNB Modulation Salvages Spectrum, Microwaves \& RF, 48 (2009)55-63.

[2] S.K. Zhang, Assessing Different Ultranarrowband Formats, Microwaves \& RF, 51(2012).

[3] J. Mitola, Cognitive radio for flexible mobile multimedia communications, in Proc. IEEE Int. Workshop on Mobile Multimedia Communications (MoMuC), San Diego, CA, (1999) 3-10.

[4] S.K Zhang, Study on the General Design Scheme of UNB Modulation Formats, Wireless Personal Communications online first (2012): 1-15.

[5] M.Schwartz, Information transmission modulation and noise, New York: McGraw-Hill, 1980: 446-450. 